\title{
Diversidad arbórea a través de gradientes topográficos en la región de El Salto, Durango
}

\author{
Arboreal diversity through topographic gradients in the El Salto, Durango Region \\ María de Jesús Medrano-Meraz ${ }^{1}$, Francisco Javier-Hernández ${ }^{2 *}$, Sacramento Corral-Rivas ${ }^{2}$, Juan Abel \\ Nájera-Luna ${ }^{2}$ \\ ${ }^{1}$ Programa de Maestría en Ciencias Forestales, Instituto Tecnológico de El Salto, Mesa del Tecnológico S/N. C.P. 34942. El \\ Salto, Durango, México. \\ ${ }^{2}$ División de Estudios de Posgrado e Investigación, Instituto Tecnológico de El Salto, Mesa del Tecnológico, S/N. C.P. 34942. \\ El Salto, Durango, México. \\ ${ }^{*}$ Autor de correspondencia: fcojhernan@yahoo.com.mx*
}

Artículo científico recibido: 02 de junio de 2016 aceptado: 01 de marzo de 2017

RESUMEN. El objetivo del estudio fue evaluar y comparar la diversidad arbórea sobre diferentes gradientes de exposición y pendiente en un rango de altitud de 1500 a $3000 \mathrm{msnm}$ en bosques templados de la región de El Salto, Durango. La base de datos se obtuvo de 268 sitios de 0.1 ha distribuidos al azar en 12 comunidades. Para cada gradiente, se estimó la riqueza de especies (S), índice de diversidad proporcional de Shannon-Wiener ( $\mathrm{H}^{\prime}$ ), índice de Simpson $(\lambda)$ e índice de equidad de Pielou (J'). La comparación de la diversidad arbórea se realizó con análisis de rarefacción. Los resultados muestran que los factores exposición y pendiente modifican la riqueza de especies y la diversidad proporcional, mientras que los índices de Shannon-Wiener y Simpson presentaron una diversidad media y la abundancia de las especies tiende a ser heterogénea. El análisis de rarefacción indicó que existen diferencias significativas en la diversidad proporcional y la riqueza de especies, en tanto que las especies con mayor índice de valor de importancia fueron Pinus durangensis y $P$. cooperi. La exposición es la fuente de variación más importante sobre la riqueza y diversidad proporcional de especies arbóreas en comparación con la pendiente.

Palabras clave: Exposición, pendiente, rarefacción, riqueza, Shannon

ABSTRACT. The objective of the study was to evaluate and compare arboreal diversity on different gradients of exposure and slope in an altitude range of 1,500 to 3,000 m.a.s.I. in temperate forests of the El Salto, Durango region. The database was obtained from 268 sites of 0.1 ha randomly distributed in 12 communities. For each gradient, the species richness (S) was considered, as well as Shannon-Wiener proportional diversity index ( $\left.\mathrm{H}^{\prime}\right)$, Simpson index $(\lambda)$ and Pielou equity index $\left(\mathrm{J}^{\prime}\right)$. The comparison of arboreal diversity was performed with rarefaction analysis. The results show that the exposure and slope factors modify species richness and proportional diversity, while the Shannon-Wiener and Simpson indexes presented an average diversity and species abundance tends to be heterogeneous. The rarefaction analysis indicated that there are significant differences in proportional diversity and species richness, while the species with the highest importance value index were Pinus durangensis and $P$. cooperi. Exposure is the most important source of variation on the richness and proportional diversity of arboreal species compared to the slope.

Key words: Exposure, slope, rarefaction, richness, Shannon

\section{INTRODUCCIÓN}

Un tema recurrente en ecología es la determinación de la contribución de los factores que influyen en la diversidad de las comunidades forestales a lo largo de gradientes del medio ambiente (Liu et al. 2014). Información que es de gran utilidad en la predicción de la presencia o ausencia de especies para su aplicación en la planeación y ejecución de las prácticas de manejo con fines de protección, conser- 
vación y restauración de las áreas forestales (RíosAltamirano et al. 2016). Los estudios de la distribución potencial de las especies a nivel paisaje en relación a las condiciones del medio ambiente se ha abordado con análisis de discrepancia (Scott y Csuti 1997), métodos BIOCLIM (Busby 1991) y ENFA (Hirzel et al. 2002) y métodos basados en inteligencia artificial como DOMAIN (Carpenter et al. 1993), GARP (Anderson et al. 2003), modelos multivariados y MAXENT (Pliscoff y Fuentes-Carrillo 2011).

La conformación de las comunidades forestales es el resultado de la contribución de factores abióticos del sitio expresados por la altitud, exposición y pendiente (Beck et al. 2007). La exposición y pendiente, al relacionarse con las condiciones microambientales y edáficas (Siles et al. 2004), juegan un papel importante en la presencia de especies y en la distribución estructural de las comunidades vegetales (Serrada 2008). Bajo ciertas condiciones de pendiente y exposición, el suelo y las copas de los árboles reciben de manera directa a diferentes horas del día mayor o menor cantidad de radiación solar, incidiendo de forma directa en la regeneración (Takyu et al. 2002), supervivencia, crecimiento y desarrollo de las especies (Bellingham y Tanner 2000). Los bosques han sido evaluados para estimar la producción forestal maderable, por medio de mediciones dasométricas y epidométricas (Castillo-López et al. 2013, Rodríguez-Carrillo et al. 2015), pero poco se han evaluado las condiciones en términos de biodiversidad y riqueza de especies que prevalecen dentro de las áreas de manejo. Por lo anterior, el objetivo del presente estudio fue analizar la influencia de la exposición y pendiente en la riqueza y diversidad de especies arbóreas, además de determinar el valor de importancia de especies.

\section{MATERIALES Y MÉTODOS}

\section{Descripción del área de estudio}

El estudio se realizó en la región de El Salto, Durango, ubicada en la parte suroeste del estado en el macizo montañoso de la Sierra Madre Occidental. La región forestal de El Salto abarca aproximadamente 507127 ha. La altura sobre el nivel del mar fluctúa entre 1400 y 3000 m (INEGI 2010). Según la clasificación climática de Köeppen adaptada para México por García (1981), los climas que predominan en el área de estudio son de tipo templado (C) con variaciones de (A)C(W2) semicálido subhúmedo, C(W2) templado subhúmedo y $C(E)(W 2)$ semifrío subhúmedo; la precipitación media anual es de $1200 \mathrm{~mm}$, con régimen de lluvias de junio a septiembre.

\section{Muestreo}

En una superficie forestal de 59602 ha, se seleccionaron 12 comunidades (Figura 1) representativas de los bosques de clima templado, en las que se distribuyeron de manera aleatoria 268 sitios circulares de 0.1 ha, cantidad suficiente de sitios para registrar todo el espectro de intervalos de pendiente, exposiciones de la pendiente y categorías de diámetros $(\geq$ a $7 \mathrm{~cm})$ y alturas $(\geq$ a $2 \mathrm{~m})$ presentes en las comunidades (Tabla 1). La pendiente, dada en porcentaje, se clasificó en intervalos de 10 $\%$ dentro de un rango de 0 a $>70 \%$, mientras que la exposición, se determinó por el rumbo magnético, en Norte, Sur, Este, Oeste, Noreste, Sureste, Noroeste, Suroeste y Zenital. La identificación de las especies conocidas se realizó en campo y cuando no fue posible, las muestras se llevaron al laboratorio para identificarlas con las claves correspondientes.

\section{Análisis de datos}

Dentro de cada gradiente de exposición y pendiente se estimó la riqueza de especies (S), índice de diversidad proporcional de Shannon-Wiener $\left(\mathrm{H}^{\prime}\right)$, índice de Simpson $(\lambda)$ e índice de equidad de Pielou $(J ')$ con las siguientes ecuaciones:

$$
\begin{aligned}
& \mathrm{H}^{\prime}=-\sum_{i=1}^{S} \text { pi } \ln (\mathrm{pi}) \\
& \lambda=1-\sum \mathrm{p}_{i}^{2} \\
& \mathrm{~J}=\mathrm{H}^{\prime} / \mathrm{H}_{\max }=\mathrm{H}^{\prime} / \operatorname{Ln}(\mathrm{S})
\end{aligned}
$$

Donde: pi es igual a la abundancia proporcional del número de individuos de cada especie $\left(\mathrm{n}_{i}\right)$ entre el 


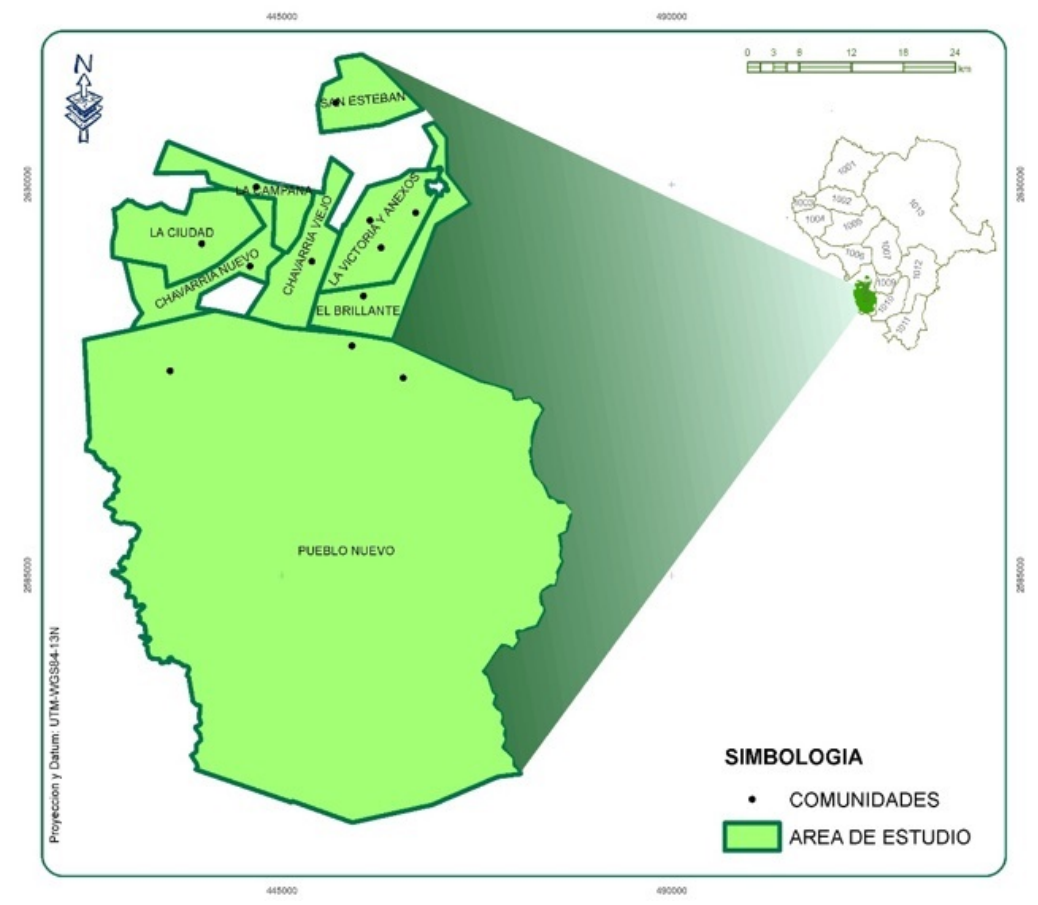

Figura 1. Localización de las comunidades muestreadas.

Tabla 1. Valores mínimos, máximos, promedio y desviación estándar de variables evaluadas en las comunidades estudiadas.

\begin{tabular}{lcccccc}
\hline \multirow{2}{*}{ Comunidades } & \multicolumn{3}{c}{ Diámetro normal $(\mathbf{c m})$} & \multicolumn{3}{c}{ Altura total $(\mathbf{m})$} \\
\cline { 2 - 7 } & Mínimo & Máximo & Promedio \pm DE & Mínimo & Máximo & Promedio \pm DE \\
\hline Chavarría Nuevo & 7 & 69 & $18.50 \pm 8.91$ & 2 & 27 & $12.03 \pm 4.86$ \\
Chavarría Viejo & 8 & 68 & $20.18 \pm 9.06$ & 3 & 27 & $11.52 \pm 4.52$ \\
La Campana & 7.5 & 67.8 & $20.41 \pm 10.23$ & 3 & 28.9 & $12.76 \pm 5.53$ \\
La Ciudad & 7.3 & 76 & $20.86 \pm 10.17$ & 3 & 35.8 & $14.64 \pm 5.47$ \\
La Peña & 8 & 77 & $28.40 \pm 13.01$ & 2 & 25 & $14.75 \pm 6.65$ \\
La Rosilla & 9.6 & 80 & $23.70 \pm 12.38$ & 2.8 & 30 & $12.13 \pm 6.01$ \\
La Victoria & 7 & 74.5 & $22.87 \pm 12.66$ & 2 & 33.7 & $11.69 \pm 5.63$ \\
Pino Gordo & 8 & 72 & $20.88 \pm 10.40$ & 3 & 25 & $12.62 \pm 5.60$ \\
Puentecillas & 7.2 & 85 & $20.65 \pm 12.48$ & 2.5 & 28 & $10.39 \pm 5.10$ \\
San Antonio & 9 & 78.5 & $24.35 \pm 13.78$ & 3.3 & 26 & $10.05 \pm 4.58$ \\
San Esteban & 7.3 & 63 & $19.22 \pm 8.95$ & 2 & 26 & $10.91 \pm 4.70$ \\
Santa Lucía & 7.5 & 63 & $19.47 \pm 10.47$ & 2 & 29 & $11.30 \pm 5.51$ \\
\hline DE= Desviación estándar & & & & & &
\end{tabular}

número total de individuos $(\mathrm{N}), \mathrm{H}_{\max }$ representa a la máxima diversidad de Shannon que es igual al logaritmo natural (In) del número de especies (S).

\section{Comparación de la diversidad}

Con el propósito de detectar diferencias significativas en la riqueza de especies, diversidad proporcional de Shannon-Wiener y Simpson e índice de equidad de Pielou entre los diferentes gradientes de pendiente y exposiciones, se empleó el método de rarefacción (Gotelli y Colwell 2011). Este método, al generar un tamaño de muestra pertinente a partir de la base de datos con el procedimiento de simulación Montecarlo, tiene la propiedad de estimar el promedio, la varianza y los intervalos de confianza de cada comunidad, estimadores esenciales para comparar la diversidad entre comunidades. Por lo que se realizaron 1000 simulaciones con el software 

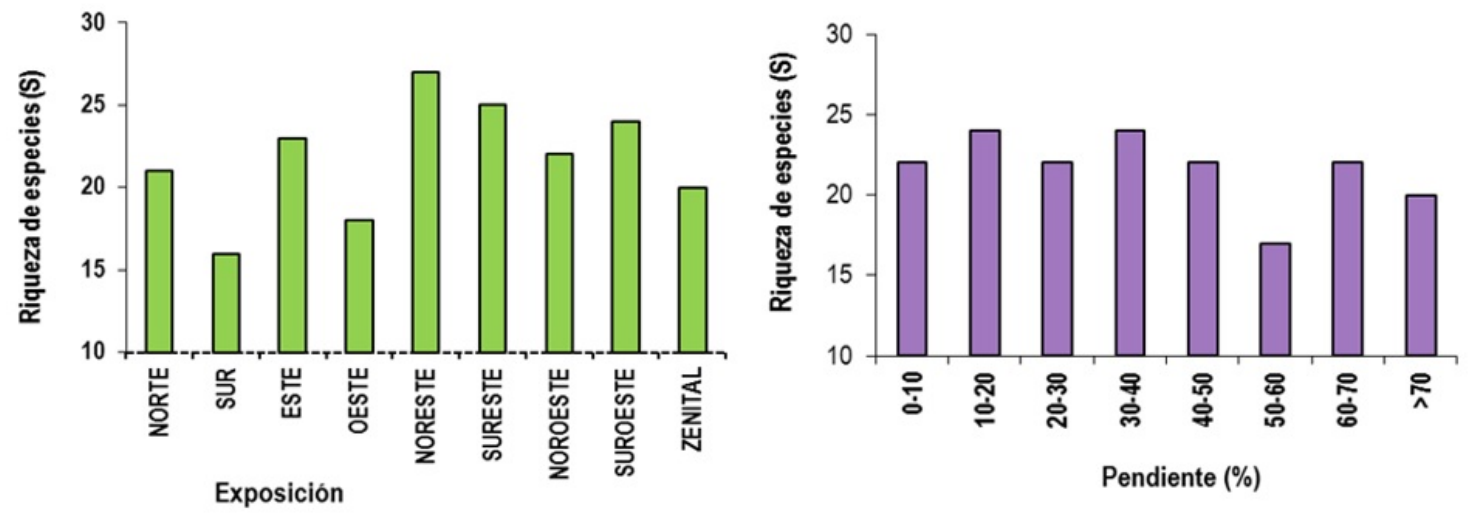

Figura 2. Riqueza de especies por exposición y pendiente.

Estimates v8.2 (Colwell 2009).

\section{Índice de valor de importancia (IVI)}

Éste es un índice desarrollado para jerarquizar la dominancia de cada especie. Se estimó el IVI de cada gradiente de exposición y pendiente, calculado con la suma de los parámetros de la estructura horizontal:

IVI = Dominancia relativa (DoR) + Densidad relativa $(\mathrm{DeR})+$ Frecuencia relativa $(\mathrm{FR})$

Considerando las siguientes ecuaciones:

$\mathrm{DoR}=\frac{\text { Dominancia absoluta por especie }}{\text { Dominancia absoluta de todas las especies }} \times 100$

Dominancia absoluta $=\frac{\text { Área basal de una especie }}{\text { Área muestreada }}$

Área Basal $=\frac{\pi}{4} \mathrm{dn}^{2}$

$\mathrm{DeR}=\frac{\text { Densidad absoluta por especie }}{\text { Densidad absoluta de todas las especies }} \times 100$

$\mathrm{DeA}=\frac{\text { Número de individuos de una especie }}{\text { Área muestreada }}$

$\mathrm{FR}=\frac{\text { Frecuencia absoluta por especie }}{\text { Frecuencia absoluta de todas las especies }} \times 100$

$\mathrm{FA}=\frac{\text { Número de sitios en los que se presenta cada especie }}{\text { Número de sitios muestreados }}$

Donde: DeA es Densidad Absoluta y FA es Frecuencia Absoluta.

\section{RESULTADOS}

\section{Especies por exposición y pendiente}

El mayor número de especies se encontró en la exposición noreste. Pero de acuerdo con la pendiente, la mayor riqueza se registró en los rangos de 10 a $20 \%$ y 30 a $40 \%$, mientras que la menor riqueza se presentó de 50 a $60 \%$ (Figura 2).

\section{Diversidad proporcional por exposición y pen- diente}

Los índices de Shannon-Wiener y Simpson, muestran que la menor diversidad de las especies arbóreas se presentó en la exposición Oeste $\left(H^{\prime}=2.09\right.$, $\lambda=0.82)$, mientras que la mayor diversidad se tuvo en las exposiciones Zenital y Norte (2.59 y $\lambda=0.90)$. Estos resultados están altamente correlacionados con el índice de equidad de Pielou, el cual muestra la existencia de una mayor homogeneidad en abundancia entre las especies en la exposición Norte $\left(J^{\prime}=0.72\right)$; en contraste, la mayor heterogeneidad se presentó en las exposiciones Oeste y Zenital $\left(\mathrm{J}^{\prime}=0.58\right)$ (Tabla 2).

De acuerdo a los índices de Shannon y Simpson, la mayor y menor diversidad de especies se encontraron en los rangos de pendiente de 60 a 70 $\%\left(H^{\prime}=2.72, \lambda=0.92\right)$ y de 40 a $60 \%\left(H^{\prime}=2.20\right.$, $\lambda=0.82)$, respectivamente. El índice de equidad de Pielou refuerza lo encontrado, al indicar que los rangos de pendiente con mayor y menor diver- 
sidad son los que presentan las abundancias más homogéneas $\left(\mathrm{J}^{\prime}=0.76\right)$ y heterogéneas $\left(\mathrm{J}^{\prime}=0.61\right)$, respectivamente.

Tabla 2. Diversidad y equidad de especies, en diferentes gradientes de exposición y pendiente en bosques de la región de El Salto, Durango.

\begin{tabular}{lccc}
\hline Exposición & $\begin{array}{c}\text { Diversidad } \\
\text { (Shannon-Wiener) }\end{array}$ & $\begin{array}{c}\text { Equidad } \\
\text { (Pielou) }\end{array}$ & $\begin{array}{c}\text { Diversidad } \\
\text { (Simpson) }\end{array}$ \\
\hline Norte & 2.59 & 0.72 & 0.90 \\
Sur & 2.22 & 0.62 & 0.86 \\
Este & 2.58 & 0.71 & 0.89 \\
Oeste & 2.09 & 0.58 & 0.82 \\
Noreste & 2.50 & 0.69 & 0.87 \\
Sureste & 2.39 & 0.66 & 0.85 \\
Noroeste & 2.28 & 0.63 & 0.84 \\
Suroeste & 2.43 & 0.67 & 0.87 \\
Zenital & 2.09 & 0.58 & 0.83 \\
\hline & Diversidad & Equidad & Diversidad \\
Pendiente & (Shannon-Wiener) & (Pielou) & (Simpson) \\
\hline $0-10 \%$ & 2.22 & 0.62 & 0.84 \\
$10-20 \%$ & 2.58 & 0.72 & 0.89 \\
$20-30 \%$ & 2.49 & 0.69 & 0.88 \\
$30-40 \%$ & 2.35 & 0.65 & 0.86 \\
$40-50 \%$ & 2.20 & 0.63 & 0.85 \\
$50-60 \%$ & 2.20 & 0.61 & 0.82 \\
$60-70 \%$ & 2.72 & 0.76 & 0.92 \\
$>70 \%$ & 2.56 & 0.71 & 0.89 \\
\hline
\end{tabular}

\section{Análisis de rarefacción}

De acuerdo al traslape de los intervalos de confianza, la riqueza de especies fue similar entre la mayoría de las exposiciones, excepto en la exposición Sur que fue similar a las exposiciones Este, Oeste, Sureste y Zenital. En relación a la diversidad proporcional de Shannon, la orientación Noreste tuvo resultados similares a las orientaciones Este, Sureste y Suroeste, mientras que la Noroeste fue similar a la Sureste y la Oeste a la Zenital. Considerando los rangos de pendiente, la riqueza de especies fue significativamente menor en los rangos de pendiente de 0 a $10 \%$ y de 50 a $60 \%$, mientras que la mayor riqueza se presentó en el rango de pendiente de 60 a $70 \%$. De acuerdo al índice de Shannon, la menor diversidad proporcional se tuvo en los intervalos de 0 a $10 \%$ y lo mayor diversidad en el intervalo de 60 a $70 \%$ (Tabla 3).

\section{Índice de valor de Importancia (IVI)}

Estructuralmente, Pinus cooperi y $P$. durangensis fueron las especies de mayor valor de importancia dentro de las exposiciones (Tabla 4) y rangos de pendiente (Tabla 5). Mientras que dentro de las exposiciones Norte, Este, Noroeste y Zenital, e intervalos de pendiente dentro del rango de 20 a $50 \%$ y de 60 a $70 \%$, la especie $P$. cooperi tuvo el mayor valor de importancia, en tanto que $P$. durangensis tuvo la mayor importancia en las exposiciones Sur, Oeste, Noreste, Sureste y Suroeste, y en las pendientes de 0 a $20 \%, 50$ a $60 \%$ y $>70 \%$. Otras especies de importancia estructural fueron $P$. strobiformis, P. leiophylla, Q. sideroxyla y Juniperus deppeana.

\section{DISCUSIÓN}

La mayor riqueza de especies se tuvo en la exposición Noreste, mientras que la menor riqueza se registró en la exposición Sur (Figura 2). Estos resultados son similares a los reportados para otros ecosistemas mexicanos (Encina-Domínguez et al. 2007, Díaz et al. 2012, Ríos-Altamirano et al. 2016) y de otras partes del mundo (Maren et al. 2015, Tavankar y Khalkhali 2015). Pero difieren de los reportados por da Silva et al. (2008) y Stage y Salas (2006), quienes mencionan que la exposición y la pendiente no influyen en la composición de especies. En particular, Altamirano et al. (2016) reportan que la mayoría de las especies de pino de Capulálpam de Méndez de la Sierra de Juárez, Oaxaca se encontraron asociadas a la exposición Noroeste, mientras que Encina-Domínguez et al. (2007) mencionan que las asociaciones de bosques de Quercus greggii-Q. mexicana de la sierra de Zapalinamé se desarrollan en laderas con exposición Norte. Debido a que la exposición Noreste propicia condiciones de temperatura y humedad favorables para el establecimiento y desarrollo de un mayor número de las especies maderables en los bosques de la región. Sobre este particular, los bosques expuestos a la exposición Noreste presentan menor déficit hídrico debido a la menor temperatura y evapotranspiración que tienen durante el día (López-Gómez et al. 2012). Las plantas establecidas en esta exposición, dentro del hemisferio norte, reciben radiación solar directa sólo en 
Tabla 3. Comparación de riqueza y diversidad de especies mediante el método de rarefacción para los niveles de exposición y pendiente evaluados.

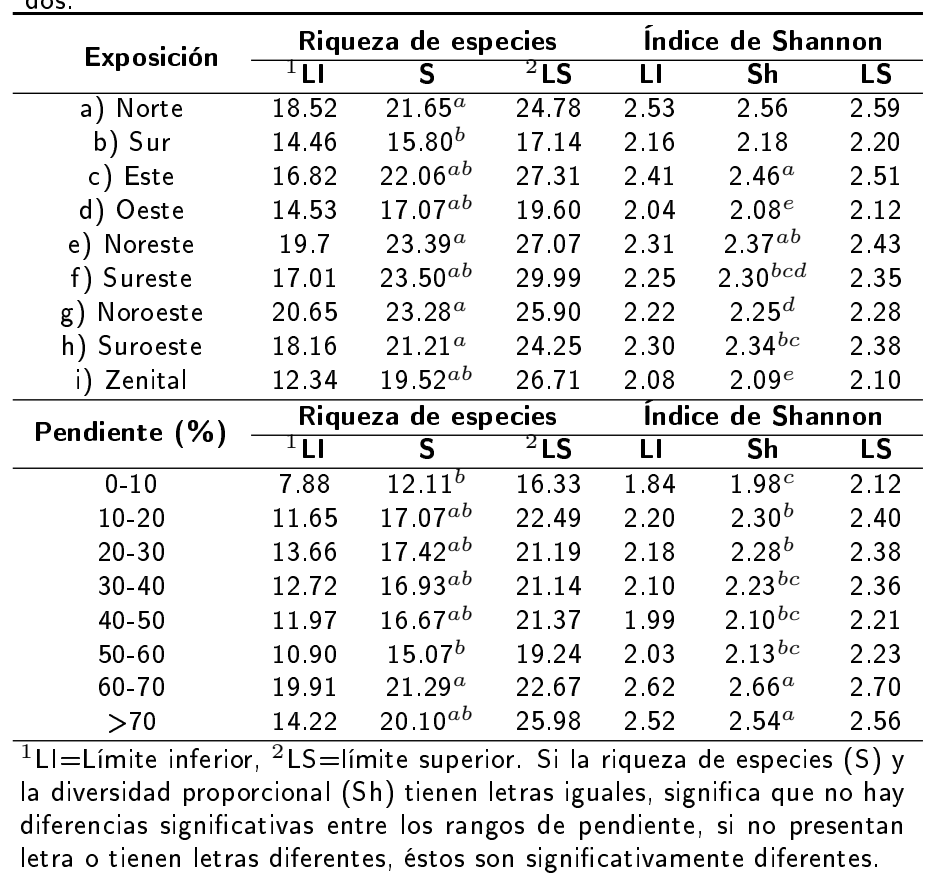

las primeras horas de la mañana, minimizando el número de horas de insolación (Serrada 2008). Al respecto Maren et al. (2015) y Ríos-Altamirano et al. (2016) mencionan que la radiación solar, temperatura y precipitación, son factores que están relacionados con la exposición, e influyen de forma positiva en la composición de especies de las áreas forestales.

Los indicadores de Shannon y Simpson indican que la exposición Norte tuvo la mayor diversidad (Tabla 2) en comparación con las otras orientaciones, presentando la orientación Oeste la menor diversidad. Los sitios con exposición Norte son más húmedos que los que tienen orientación Sur (Mazzola et al. 2008), lo cual podría explicar a los resultados encontrados en los índices de diversidad. En tanto que en las áreas orientadas al Norte, la temperatura es menor, y la precipitación y los niveles de humedad del suelo son mayores, mientras que en las áreas orientadas al Sur las condiciones son más cálidas con menor de humedad (Allen et al. 1991). Por otro lado, aunque las orientaciones hacia el Oeste deben de recibir mayor cantidad de luz durante la tarde, ésta es inhibida por la presencia de nubosidad en horas vespertinas (Torres et al. 2012), lo que restringe la presencia de especies y fomenta la dominancia de otras. Aunque la pendiente está identificada como una variable indicadora del potencial de los recursos hidrícos y nutrimentales del suelo que influye en la distribución de la composición de especies (Tilman y Pacala 1993), en este estudio, la diversidad arbórea no tuvo una tendencia definida en función de la pendiente (Figura 2). Lo que se puede deber a que los factores ambientales relacionados con la exposición tienen mayor influencia que las condiciones del suelo en el establecimiento de las especies. Al respecto Quinto-Mosquera y MorenoHurtado (2014), mencionan que la diversidad de especies esta limitada por las condiciones de productividad a nivel local.

La diversidad muestra que las comunidades forestales presentan una diversidad media en todos los niveles de exposición y pendiente estudiados. De acuerdo a Margalef (1972), el índice de Shannon normalmente varía de 1 a 5 , siendo valores menores de 2 característicos de diversidad baja, de 2 a 3.5 
Tabla 4. Índice de Valor de Importancia de las especies presentes en las diferentes exposiciones.

\begin{tabular}{|c|c|c|c|c|c|c|c|c|c|}
\hline \multirow{2}{*}{ Especie } & \multicolumn{9}{|c|}{ Exposiciones } \\
\hline & Norte & Sur & Este & Oeste & Noreste & Sureste & Noroeste & Suroeste & Zenital \\
\hline Alnus jorullensis & 2.70 & $*$ & 2.38 & $*$ & 3.42 & 1.45 & 1.57 & 1.70 & 2.39 \\
\hline Juniperus deppeana & 18.22 & 22.65 & 20.68 & 20.30 & 17.02 & 17.04 & 15.58 & 15.37 & 24.53 \\
\hline Arbutus xalapensis & $*$ & 2.25 & $*$ & $*$ & 2.44 & 1.29 & 7.07 & 2.47 & 2.22 \\
\hline Arbutus chapensis & 14.82 & 14.43 & 14.47 & 8.19 & 12.03 & 13.68 & 4.83 & 13.4 & 14.54 \\
\hline Quercus candicans & $*$ & $*$ & 2.54 & 2.18 & 2.31 & 1.67 & 2.20 & 3.39 & 4.40 \\
\hline Quercus castanea & $*$ & * & $*$ & $*$ & 1.03 & $*$ & $*$ & $*$ & $*$ \\
\hline Quercus crassifolia & 13.95 & 6.78 & 5.41 & 1.84 & 5.86 & 5.3 & * & 5.21 & 2.08 \\
\hline Quercus conzattii & 3.44 & $*$ & 2.23 & $*$ & 1.10 & 6.49 & 3.63 & 6.01 & $*$ \\
\hline Quercus durifolia & 5.34 & 3.10 & 5.14 & 2.13 & 3.32 & 3.13 & $*$ & 3.67 & 2.15 \\
\hline Quercus eduardii & $*$ & 2.44 & 1.31 & & 2.06 & 2.16 & $*$ & $*$ & 2.22 \\
\hline Quercus fulva & $*$ & $*$ & * & $*$ & $*$ & $*$ & 2.06 & 1.91 & * \\
\hline Quercus rugosa & 15.3 & 17.20 & 13.11 & 14.76 & 16.92 & 14.89 & 10.14 & 16.04 & 13.51 \\
\hline Quercus sideroxyla & 32.55 & 40.45 & 33.9 & 33.62 & 34.86 & 30.25 & 39.28 & 47.83 & 45.5 \\
\hline Quercus urbanii & 1.68 & $*$ & $*$ & 1.87 & 3.14 & 3.38 & 2.34 & $*$ & $*$ \\
\hline Quercus viminea & $*$ & * & 2.17 & $*$ & $*$ & 1.55 & $*$ & * & 4.16 \\
\hline Pinus strobiformis & 27.74 & 22.75 & 20.6 & 20.49 & 22.30 & 13.45 & 18.76 & 20.86 & 24.1 \\
\hline Pinus chihuahuana & 3.51 & $*$ & 2.9 & 7.04 & 1.39 & 1.85 & 3.67 & 2.52 & 2.15 \\
\hline Pinus cooperi & 51.59 & 47.33 & 47.47 & 61.41 & 41.32 & 48.55 & 76.08 & 41.91 & 63.12 \\
\hline Pinus douglasiana & 7.59 & $*$ & 6.29 & $*$ & 2.07 & 2.77 & 6.36 & 2.19 & $*$ \\
\hline Pinus durangensis & 26.34 & 62.38 & 47.65 & 70.83 & 60.46 & 63.96 & 43.51 & 53.95 & 57.37 \\
\hline Pinus engelmannii & 22.06 & $*$ & 13.82 & 10.20 & 7.80 & 9.73 & 14.44 & 12.83 & 2.18 \\
\hline Pinus herrerae & 18.62 & 7.13 & 6.22 & 4.06 & 6.47 & 6.30 & 6.98 & 8.79 & $*$ \\
\hline Pinus leiophylla & 18.71 & 20.66 & 10.12 & 12.46 & 19.02 & 16.22 & 13.14 & 12.12 & 12.66 \\
\hline Pinus lumholtzii & 5.36 & 5.67 & 8.18 & 10.4 & 8.08 & 12.92 & 8.80 & 4.35 & 4.54 \\
\hline Pinus michoacana & 6.84 & 4.94 & 9.78 & 4.32 & 5.82 & 4.41 & 2.65 & 4.38 & $*$ \\
\hline Pinus teocote & 1.66 & 19.86 & 12.58 & 13.92 & 13.78 & 10.15 & 11.66 & 15.31 & 14.09 \\
\hline Pinus oocarpa & 1.99 & $*$ & 11.05 & $*$ & 2.87 & 7.42 & 5.25 & 2.41 & 2.09 \\
\hline Pseudotsuga menziesii & * & $*$ & $*$ & * & 2.20 & $*$ & $*$ & $*$ & $*$ \\
\hline Abies durangensis & * & * & * & * & $*$ & * & * & 1.37 & * \\
\hline Populus tremuloides & * & * & * & * & * & * & * & * & 0.93 \\
\hline
\end{tabular}

diversidad media, y mayores a 3.5 de diversidad alta. De acuerdo al análisis de rarefacción, la riqueza de especies y la diversidad proporcional mostraron diferencias estadísticamente significativas entre algunos rangos de pendiente y exposiciones. El índice de valor de importancia (IVI) es un indicador estructural, cuantitativo y preciso, desarrollado para jerarquizar la dominancia e importancia de cada especie, considerando su abundancia, cobertura y presencia espacial dentro de la comunidad (Zarco-Espinoza et al. 2010). Al respecto, Campo y Duval (2014), manifiestan que las especies que presentan un valor mayor, son aquellas que poseen un patrón regular, mientras que aquellas con un valor bajo son características de un patrón irregular disperso. En el presente estudio, las especies arbóreas con mayor dominancia y distribución espacial homogénea fueron $P$. durangensis y $P$. cooperi, siguiendo en orden de importancia $P$. strobiformis, P. leiophylla, Q. sidroxyla, Q. rugosa, Juniperus deppeana y Arbutus chapensis, especies con presencia en todos los gradientes evaluados. Los menores índices de valor de importancia los presentaron Pseutotsuga menziesii, Populus tremuloides y Abies durangensis, lo que reafirma la preferencia de estas especies por hábitats exclusivos dentro de la región (González-Elizondo et al. 2012). La especie $P$. menziesii tuvo presencia en la exposición Noreste con pendientes superiores a $70 \%$, mientras que Abies durangensis en la exposición Suroeste en pendientes de 20 a $30 \%$, P. tremuloides se detectó en áreas con pendientes mínima. Dentro del género Pinus, $P$. durangenesis es una de las especies con mayor distribución en la parte norte de la Sierra Madre Occidental del estado de Durango, debido a su buena adaptabilidad a los diferentes gradientes topográficos (González et al. 2007). 
Tabla 5. Índice de Valor de Importancia en los rangos de pendiente evaluados.

\begin{tabular}{|c|c|c|c|c|c|c|c|c|}
\hline \multirow{2}{*}{ Especie } & \multicolumn{8}{|c|}{ Rangos de pendiente (\%) } \\
\hline & $0-10$ & $10-20$ & $20-30$ & $30-40$ & $40-50$ & $50-60$ & $60-70$ & $>70$ \\
\hline Alnus jorullensis & 2.70 & 2.54 & * & 1.48 & 1.34 & * & * & 4.78 \\
\hline Juniperus deppeana & 23.30 & 13.93 & 19.34 & 23.70 & 17.91 & 7.53 & 3.37 & 3.56 \\
\hline Arbutus xalapensis & 1.29 & 3.33 & & 2.84 & 3.64 & 2.23 & 3.40 & 4.62 \\
\hline Arbutus chapensis & 12.30 & 13.66 & 6.99 & 11.95 & 13.08 & 11.58 & 10.86 & 26.73 \\
\hline Quercus candicans & * & 1.09 & * & 5.07 & $*$ & 6.95 & 11.51 & 18.30 \\
\hline Quercus castanea & * & * & * & $*$ & 1.40 & $*$ & * & * \\
\hline Quercus crassifolia & 8.00 & 4.59 & 2.78 & 1.44 & 4.73 & * & * & 8.55 \\
\hline Quercus conzattii & 3.67 & 3.19 & 1.32 & 1.56 & 3.24 & * & 14.91 & \\
\hline Quercus durifolia & 2.26 & 1.40 & 3.00 & 1.34 & 6.43 & 8.12 & 2.73 & 16.49 \\
\hline Quercus eduardii & * & 1.94 & 1.94 & 1.65 & 1.65 & $*$ & 3.91 & * \\
\hline Quercus fulva & $*$ & * & $*$ & 2.46 & * & $*$ & * & 5.02 \\
\hline Quercus rugosa & 10.70 & 19.00 & 16.51 & 17.45 & 15.16 & 23.71 & 25.37 & 26.97 \\
\hline Quercus sideroxyla & 35.59 & 36.88 & 40.04 & 43.21 & 40.52 & 43.66 & 33.22 & 14.92 \\
\hline Quercus urbanii & $*$ & 2.86 & 2.55 & $*$ & 1.50 & 3.12 & * & * \\
\hline Quercus viminea & * & $*$ & $*$ & 2.90 & $*$ & $*$ & 12.07 & * \\
\hline Pinus strobiformis & 22.05 & 16.55 & 21.25 & 30.41 & 21.24 & 20.12 & 14.88 & 12.04 \\
\hline Pinus chihuahuana & 4.10 & 1.59 & 5.44 & $*$ & $*$ & $*$ & 6.37 & $*$ \\
\hline Pinus cooperi & 49.24 & 45.70 & 62.68 & 67.91 & 58.58 & 22.93 & 31.16 & 13.01 \\
\hline Pinus douglasiana & 1.17 & 2.51 & 8.03 & 4.97 & $*$ & 6.13 & 10.24 & 9.27 \\
\hline Pinus durangensis & 69.80 & 52.28 & 33.93 & 26.31 & 56.39 & 90.29 & 17.22 & 43.19 \\
\hline Pinus engelmannii & 4.73 & 15.11 & 14.78 & 6.42 & 2.68 & 12.30 & 32.73 & 42.23 \\
\hline Pinus herrerae & 4.96 & 13.68 & 9.04 & 8.23 & 5.06 & 2.04 & 5.67 & 14.19 \\
\hline Pinus leiophylla & 16.70 & 16.13 & 12.92 & 15.48 & 16.55 & 23.87 & 10.98 & 14.32 \\
\hline Pinus lumholtzii & 2.76 & 8.38 & 14.85 & 9.03 & 7.01 & 6.92 & 26.48 & $*$ \\
\hline Pinus michoacana & 2.21 & 7.98 & 6.52 & 4.17 & 7.40 & $*$ & 10.73 & 3.34 \\
\hline Pinus teocote & 18.21 & 10.96 & 8.50 & 6.04 & 11.63 & 8.49 & 8.77 & * \\
\hline Pinus oocarpa & 3.23 & 4.71 & 6.30 & 3.97 & 2.86 & * & 3.43 & 5.95 \\
\hline Pseudotsuga menziesii & * & * & $*$ & $*$ & * & * & $*$ & 12.52 \\
\hline Abies durangensis & * & * & 1.30 & * & * & * & * & $*$ \\
\hline Populus tremuloides & 1.05 & * & * & * & * & * & * & * \\
\hline
\end{tabular}

\section{CONCLUSIONES}

La riqueza específica varió de 14 a 25 especies, mientras que la diversidad proporcional de la exposición y pendiente fue de 2.09 a 2.72. La riqueza de especies y la diversidad proporcional fue significativamente diferente entre los diferentes rangos de exposición y pendiente. Las especies con mayor valor de importancia ( $P$. durangensis y $P$. Cooperi) se distribuyen en toda el área, en contraste, $P$. menziesii, A. durangensis y $P$. tremuloides se presentaron en áreas exclusivas.

\section{LITERATURA CITADA}

Allen RB, Reif A, Hall GMJ (1991) Elevational distribution of conifer broadleaved hardwood forests on South Island, New Zealand. Journal Vegetation Science 2: 323-330.

Anderson RP, Lew D, Townsend PA (2003) Evaluating predictive models of species' distributions: criteria for selecting optimal models. Ecological Modelling 162: 211-232.

Beck SG, Hernandez PA, Jørgensen PM, Paniagua L, Timaná ME, Young YBE (2007) Plantas basculares. En: Young BE (Ed.). Distribución de las especies endémicas en la vertiente oriental de los Andes en Perú y Bolivia. NatureServe. Virginia, USA. pp: 18-34.

Bellingham PJ, Tanner, JEV (2000) The influence of topography on tree growth, mortality, and recruitment in a tropical montane forest. Biotropica 32: 378-384. 
Busby JR (1991) BIOCLIM: A bioclimate analysis and prediction system. Plant Protection Quarterly 6: 64-68.

Campo MA, Duval VS (2014) Diversidad y valor de importancia para la conservación de la vegetación natural. Parque Nacional Lihué Calel (Argentina). Anales de Geografía 34: 25-42.

Carpenter G, Gillison AN, Winter J (1993) DOMAIN: A flexible modelling procedure for mapping potential distributions of plants and animals. Biodiversity and Conservation 2: 667-680.

Castillo-López A, Vargas-Larreta B, Corral-Rivas JJ, Nájera-Luna JA, Cruz-Cobos F, Hernández FJ (2013) Modelos compatible altura-índice de sitio para cuatro especies de pino en Santiago Papasquiaro, Durango. Revista Mexicana de Ciencias Forestales 4: 87-103.

Colwell RK (2009) Estimates 8.2 User's guide. Department of Ecology and Evolutionary Biology. University of Connecticut, USA. 45p.

da Silva WG, Metzger JP, Bernacci LC, Martins-Catharino LE, Durigan G, Simoes S (2008) Relief influence on tree species richness in secondary forest fragments of Atlantic forest, SE, Brazil. Acta Botánica Brasilica 22: 589-598.

Díaz V, Sosa-Ramírez J, Pérez-Salicrup DR (2012) Distribución y abundancia de las especies arbóreas y arbustivas de la Sierra Fría, Aguascalientes, México. Polibotánica 34: 99-126.

Encina-Domínguez JA, Zarate-Lupercio A, Valdéz-Reyna J, Villarreal-Quintanilla JA (2007) Caracterización ecológica y diversidad de los bosques de encino de la Sierra de Zapalinamé, Coahuila, México. Boletín de la Sociedad Botánica de México 81: 51-63.

García ME (1981) Modificaciones al sistema de clasificación climática de Köppen. Editado por la autora. México. 252p.

González EMS, González EM, Márquez LMA (2007) Vegetación y Ecorregiones de Durango. Ed. Plaza y Valdez editores. México. 219p.

González-Elizondo MS, González-Elizondo M, Tena-Flores JA, Ruacho-González L, López-Enríquez IL (2012) Vegetación de la Sierra Madre Occidental México: una síntesis. Acta Botánica Mexicana 100: 351-403.

Gotelli NJ, Colwell RK (2011) Estimating species richness. In: Magurran AE, McGill BJ (Ed.). Biological diversity: Frontiers in measurement and assessment. Oxford University Press. Oxford, EUA. pp: 39-54.

Hirzel AH, Hausser,J, Chessel D, Perrin N (2002) Análisis ecológico de factores de nicho: ¿Cómo calcular mapas de aptitud para el hábitat sin datos de ausencia? Ecología 83: 2027-2036.

INEGI (2010) Instituto Nacional de Estadística y Geografía. http://www.beta.inegi.org.mx/app/mapa/espacioydatos/default.aspx?o=1008858001. Fecha de consulta 5 de agosto de 2015.

Liu J, Yunhong T, Ferry-Silk JW (2014) Topography related habitat associations of tree species traits, composition and diversity in Chinese tropical forest. Forest Ecology and Management 330: 75-81.

López-Gómez V, Zedillo-Avelleyra P, Anaya-Hong S, González-Lozada E, Cano-Santana Z (2012) Efecto de la orientación de la ladera sobre la estructura poblacional y ecomorfología de Neobuxbaumia tetetzo (Cactaceae). Botanical Sciences 90: 453-457.

Maren IE, Karki S, Prajapati Ch, Yadav RK, Shrestha BB (2015) Phasing north or south: Does slope aspect impact forest stand characteristics and soil properties in a semiarid trans-Himalayan valley? Journal of Arid Environments 121: 112-123. 
Margalef R (1972) Homage to E. Hutchison, or why is there an upper limit to diversity. Transactions of the Connecticut Academy of Arts and Sciences 44: 211-235.

Mazzola MB, Kin AG, Morici EF, Babinec FJ, Tamborini G (2008) Efecto del gradiente altitudinal sobre la vegetación de las sierras Lihue Calel (La Pama, Argentina). Boletín de la Sociedad Argentina de Botánica 43: 103-119.

Pliscoff P, Fuentes-Castillo T (2011) Modelación de la distribución de especies y ecosistemas en el tiempo y en el espacio: una revisión de las nuevas herramientas y enfoques disponibles. Revista de Geografía Norte Grande 48: 61-79.

Quinto-Mosquera H, Moreno-Hurtado F (2014) Diversidad florística arbórea y su relación con el suelo en un bosque pluvial tropical del Chocó Biogeográfico. Revista Árvore Vicosa-MG 38: 1123-1132.

Ríos-Altamirano A, Alfonso-Corrado C, Aguirre-Hidalgo V, Ángeles-Pérez G, Mendoza-Díaz MM, RodríguezRivera V, et al (2016) Abundancia y Distribución del género Pinus de Capulálpam de Méndez, Sierra Juárez, Oaxaca. Madera y Bosques 22: 61-74.

Rodríguez-Carrillo A, Cruz-Cobos F, Vargas Larreta B, Hernández FJ (2015) Compatible dominant heightsite index model for juniper (Juniperus depeana Steud.). Revista Chapingo, Serie Ciencias Forestales y del Ambiente 21: 97-108.

Scott JM, Csuti B (1997) Gap analysis for biodiversity survey and maintenance. In: Reaka-Kudla ML, Wilson DE, Wilson EO (ed.) Biodiversity II: Understanding and Protecting our Biological Resources. USA. pp: 321-340.

Serrada HR (2008) Apuntes de Selvicultura. Universidad Politécnica de Madrid. EUI Técnica Forestal. Madrid. 490p.

Siles G, Bastida J, Rey PJ, Alcántara J (2004) Modelos predictivos de distribución de especies de vegetación potencial en el incendio del puerto de las palomas "Sierra de Cazorla" Jaén. Cuadernos de la Sociedad Española de Ciencias Forestales 20: 167-172.

Stage AR, Salas Ch (2007) Interaction of elevation, aspect and slope in models of forest species composition and productivity. Forest Science 53: 486-492.

Takyu M, Aiba SI, Kitayama K (2002) Effects of topography on tropical lower montane forests under different geological conditions on Mount Kinabalu, Borneo. Plant Ecology 159: 35-49.

Tavankar F, Khalkhali MN (2015) Effect of slope aspect on woody species diversity and stand structure in mountain Hyrcanian forest. Scholars Academic Journal Biosciences 2: 151-156.

Tilman D, Pacala S (1993) The maintenance of species diversity in plant communities. In: Ricklefs RE, Schluter D (eds.) Species diversity in ecological communities. University of Chicago Press. Chicago. USA. pp: $13-25$.

Torres E, Schwarzkopf T, Fariñas M, Aranguren A (2012) ¿Es la orientación de la pendiente un factor modificador de la estructura florística en la alta montaña tropical andina?. Ecotrópicos 25: 61-74.

Zarco-Espinoza VM, Valdez-Hernández JI, Ángeles-Pérez G, Castillo-Acosta O (2010) Estructura y diversidad de la vegetación arbórea del Parque Estatal Agua Blanca, Macuspana, Tabasco. Universidad y Ciencia 26: $1-17$. 